\title{
Use of home sleep studies for diagnosis of the sleep apnoea/hypopnoea syndrome
}

\author{
A T Whittle, S P Finch, I L Mortimore, T W MacKay, N J Douglas
}

\begin{abstract}
Background - A study was undertaken to test the hypothesis that unsupervised domiciliary limited sleep studies do not impair the accuracy of diagnosis when used to investigate the sleep apnoea/hypopnoea syndrome (SAHS) and can be cheaper than laboratory polysomnography.

Methods - For validation, 23 subjects with suspected SAHS underwent laboratory polysomnography and a home study (EdenTec 3711) on successive nights. All subjects with $>15$ apnoeas + hypopnoeas

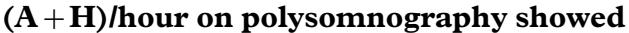
$>30 \mathrm{~A}+\mathrm{H} / \mathrm{h}$ our on their home study. Thereafter, in a prospective trial 150 subjects had a home study as the initial investigation and studies showing $>\mathbf{3 0}$ events/hour were regarded as diagnostic of SAHS. Those showing fewer events were investigated with polysomnography if necessary. Time to treatment, outcome, and costs of this protocol were compared with those of 75 patients investigated initially with polysomnography.
\end{abstract}

Results - Of the prospective trial subjects, $29 \%$ had $>30 \mathrm{~A}+\mathrm{H} / \mathrm{hour}$ and proceeded directly from home study to treatment; $15 \%$ without daytime sleepiness were not investigated further. Polysomnography was undertaken to establish a diagnosis in $56 \%$ of cases, including $18 \%$ whose home studies were unsuccessful. Compared with the 75 control patients, this protocol gave a diagnosis faster (median 18 (range 0-221) versus $47(0-227)$ days, $p<0.001)$ and more cheaply (mean (SD) $£ 164$ (104) versus $£ 210$ $(0), p<0.001)$. The proportions offered CPAP $(61 \%$ versus $67 \%)$ and subsequent objective CPAP usage (mean 4.7 (2.4) versus 5.0 (2.4) hours/night) were not different.

Conclusions - Use of home sleep studies has benefits in time and cost. For diagnostic reliability a further sleep study was required in $56 \%$ of cases.

(Thorax 1997;52:1068-1073)

Keywords: sleep apnoea/hypopnoea syndrome, home sleep studies, polysomnography.

The alleged gold standard investigation for the diagnosis of the sleep apnoea/hypopnoea syndrome (SAHS) is inpatient overnight polysomnography ${ }^{1}$ and this is recommended as standard by American ${ }^{2}$ and Australasian ${ }^{3}$ guidelines. However, SAHS may occur in up to $4 \%$ of the middle aged male population ${ }^{4}$ and, as awareness increases, the number of patients referred for investigation is rising rapidly. New referrals to the Edinburgh sleep clinic increased 10-fold between 1990 and 1996 . Most require some form of sleep study for diagnosis, and this puts increasing strain on a service based on overnight studies in the laboratory. We have therefore investigated the validity and practicality of performing limited sleep studies unsupervised in the patients' homes.

A number of portable sleep study systems have been validated by recording simultaneously with polysomnography in a sleep laboratory. ${ }^{5-11}$ Portable systems have been used for epidemiological studies ${ }^{12-14}$ and are increasingly used routinely for diagnosis or exclusion of SAHS by sleep services. ${ }^{15}{ }^{16}$ However, sleep quality is not recorded in a limited home study; subjects may sleep better at home than in a laboratory study ${ }^{17}$ but, equally, patients with severe SAHS may sleep very little. It is therefore important that the use of portable systems in the home is validated by comparing studies performed at home with polysomnography. Few of the groups using home studies have reported this; one group found that the portable system underestimated the severity of SAHS. ${ }^{18}$

Our study had two aims. The first was to validate the use of limited sleep studies in the home, and to determine a criterion for a confident diagnosis of SAHS from a home study. The second was to test in a prospective trial whether home studies in clinical practice can maintain diagnostic accuracy and have benefits in time and cost without affecting outcome when compared with an investigative protocol based on laboratory polysomnography. For home sleep studies we used the EdenTrace system which has been validated for laboratory use by simultaneous recording with laboratory polysomnography ${ }^{5}$ and is in clinical use in several centres. ${ }^{1516}$

\section{Methods}

SUBJECTS

The subjects were patients newly referred to our sleep clinic for investigation of suspected SAHS between February 1994 and July 1996 who lived within 30 miles of our laboratory. All subjects were judged by NJD or TWM to require a sleep study as an initial investigation on the basis of at least two major symptoms of SAHS. ${ }^{19}$ Patients were excluded from the trial if they were judged to be physically or mentally incapable of using portable sleep study equipment unsupervised and no family assistance was available, or if there was a clinical suspicion 
of cataplexy or periodic leg movement disorder. Control patients were new patients living $>30$ miles from the laboratory who attended the same initial referral clinics as the subjects for the prospective trial (one control selected for every two trial patients at a clinic) and who followed our normal investigation protocol of laboratory polysomnography booked at the time of the clinic. Data from control patients were used for comparison of costs, duration of investigation, and outcome.

VALIDATION TRIAL

Subjects underwent inpatient polysomnography on night 1 . The next morning a trained sleep technician educated them in the use of the portable sleep study system which they took home to record themselves unsupervised on night 2 and then returned the next day for analysis.

PROSPECTIVE TRIAI

The diagnostic criteria used in this protocol were based on the results of the validation trial (see results below). Subjects underwent a home study as an initial investigation after education in the use of the equipment. Home studies showing $>30$ apnoeas + hypopnoeas $(\mathrm{A}+\mathrm{H})$ per hour of recording were regarded as diagnostic of SAHS and these subjects proceeded to treatment without further investigation. Subjects whose home studies showed $<30 \mathrm{~A}+\mathrm{H}$ per hour and who denied daytime sleepiness were not investigated further but were given appropriate advice. The remaining subjects, including those in whom the home study was unsuccessful, were further investigated with overnight polysomnography in the laboratory and managed thereafter according to our usual practice. Bookings for polysomnography were given the same priority as those for control and non-trial patients.

\section{POLYSOMNOGRAPHY}

Overnight laboratory polysomnography recorded an electroencephalogram (two channels), electro-oculogram (four channels), submental electromyogram, bilateral anterior tibial electromyogram, electrocardiogram, summed oronasal airflow by thermocouple, thoracic and abdominal movement by inductive plethysmography, and ear pulse oximetry. Data were collected on a computerised recording system (Compumedics, Melbourne, Australia) but all interpretation and scoring was performed manually by experienced technicians. Sleep was scored on screen by conventional criteria. ${ }^{20}$ Apnoea was defined as the cessation of airflow for $>10$ seconds, and hypopnoea as a reduction of $>50 \%$ in thoracoabdominal movement for $>10$ seconds. ${ }^{21}$

HOME SLEEP STUDIES

The EdenTrace system (EdenTec 3711, Nellcor, Eden Prairie, Minnesota, USA) records oronasal airflow by thermocouple, electrocardiogram, chest wall movement by electrical impedance, and finger pulse oximetry. Studies were transferred to paper and scored manually. Apnoea was defined as a cessation of airflow for $>10$ seconds and hypopnoea was defined as a reduction of $>50 \%$ in chest wall movement for $>10$ seconds. Frequencies of events were calculated per hour of recording. Studies without four hours of interpretable recording were regarded as technically unsatisfactory.

\section{OUTCOMES}

All subjects or controls who later attended for laboratory titration for nasal continuous positive airway pressure therapy (CPAP) were classified as having been offered CPAP therapy. Those still in possession of a CPAP system in November 1996 (4-20 months after diagnosis) were classified as continuing CPAP. Objective CPAP usage was calculated from the number of hours running time registered on the CPAP unit's in-built time clock documented at the most recent clinic attendance, averaged over the number of days since the previous recording (median 94 days, range 10-372).

The cost of inpatient polysomnographic studies and home studies included capital depreciation of equipment, disposable equipment, medical, technician and nursing time. The cost of overnight hospital stay was included in the cost of the laboratory studies. For the portable system we assumed 1000 nights' use before replacement and allowed travel expenses for the patient's additional journey to return the equipment.

Time to diagnosis was the number of days between the clinic attendance when the decision was taken to investigate with a sleep study, and the date of the study which provided a diagnosis on which management could be based. Costs and times for subjects in the prospective protocol who underwent two sleep studies allow for both studies.

\section{STATISTICAL ANALYSIS}

The two forms of sleep study were compared in the validation trial by the method of Bland and Altman for the assessment of agreement between methods of clinical measurement. ${ }^{22}$ Normally distributed values are quoted as means with standard deviation, and the significance of differences was assessed by the two-tailed Student's $t$ test. Other values are quoted as medians with range and compared with the two-tailed Mann-Whitney U test. Where more than one set of variables was compared with control values (tables 1 and 4) the probabilities of significance have been adjusted by the Bonferroni technique. Correlations between non-parametric data are quoted as Spearman's rank correlation coefficients, and category comparisons were made with the $\chi^{2}$ test. 


\section{Results}

VALIDATION TRIAL

Twenty three subjects (19 men) of mean (SD) age 50 (9) years and mean (SD) body mass index (BMI) $30(4) \mathrm{kg} / \mathrm{m}^{2}$ completed the protocol. Twenty of the 23 home studies produced
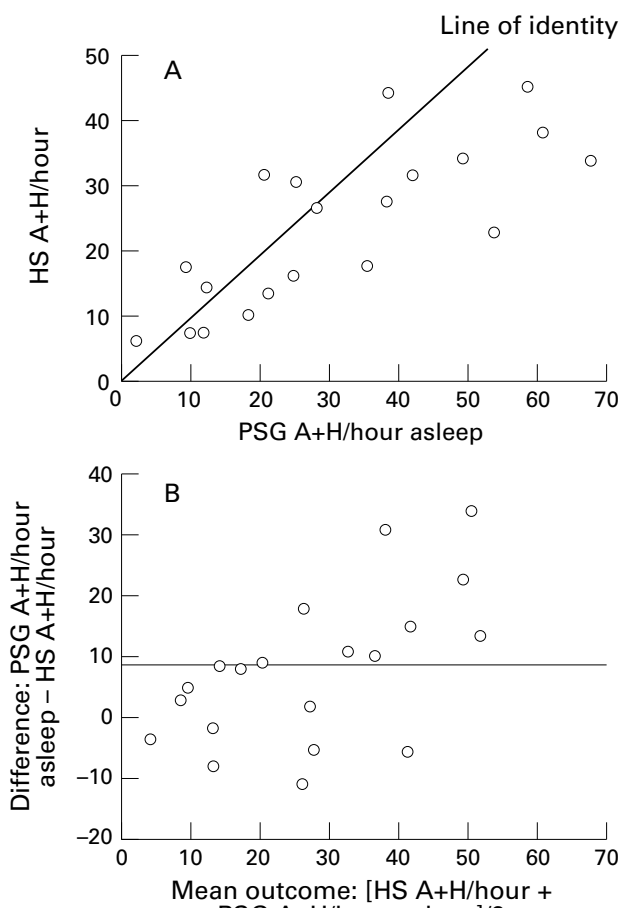

$\mathrm{PSG} \mathrm{A}+\mathrm{H} /$ hour asleep]/2

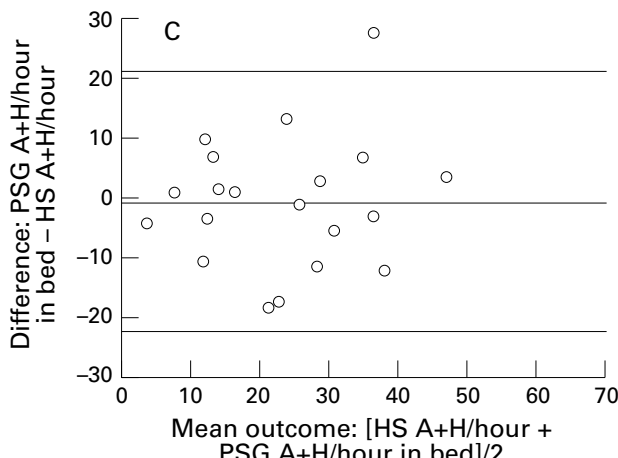

Figure 1 Validation trial. (A) Home sleep studies (HS) compared with polysomnographic (PSG)

apnoeas + hypopnoeas $(A+H) /$ hour asleep: raw data.

(B) Home sleep studies compared with polysomnographic

$A+H / h o u r$ asleep: Bland-Altman plot of difference

against mean value. Horizontal line is the mean difference. (C) Home sleep studies compared with polysomnographic $A+H / h o u r$ in bed: Bland-Altman plot of difference against mean value. Horizontal lines are mean difference and limits of agreement at the 95\% level $(m e a n \pm 1.96 S D)$

Table 1 Characteristics of control patients, prospective trial subjects, and the subset of subjects given CPAP titration following a home study as their sole investigation

\begin{tabular}{llll}
\hline & $\begin{array}{l}\text { Control patients } \\
(n=75)\end{array}$ & $\begin{array}{l}\text { Subjects (all) } \\
(n=150)\end{array}$ & $\begin{array}{l}\text { Subjects (direct to CPAP) } \\
(n=40)\end{array}$ \\
\hline Mean (SD) age (years) & $49(14)$ & $\begin{array}{l}48(13) \\
(\mathrm{p}>0.5)\end{array}$ & $\begin{array}{l}50(13) \\
(\mathrm{p}>0.5)\end{array}$ \\
Sex (\% female) & $25 \%$ & $\begin{array}{l}13 \% \\
(\mathrm{p}=0.03)\end{array}$ & $\begin{array}{l}8 \% \\
(\mathrm{p}=0.04)\end{array}$ \\
Mean (SD) BMI $\left(\mathrm{kg} / \mathrm{m}^{2}\right)$ & $33(10)$ & $\begin{array}{l}31(5) \\
(\mathrm{p}=0.1)\end{array}$ & $\begin{array}{l}32 \\
(\mathrm{p}>0.5)\end{array}$ \\
\hline
\end{tabular}

Significances refer to differences from control group. interpretable recordings. All polysomnographic studies were interpretable.

The 20 home studies showed a median of $25 \mathrm{~A}+\mathrm{H} /$ hour of recording (range 6-45). Polysomnography showed a median of $27 \mathrm{~A}+\mathrm{H} /$ hour of sleep (range 2-67) (fig 1A). The mean (SD) difference between these scores (polysomnographic minus home study) was +8 (12) A Bland-Altman plot $^{22}$ comparing the difference between each pair of results with the mean value of each pair (fig 1B) showed a bias to lower scores on home studies at higher $\mathrm{A}+\mathrm{H}$ values. The home study $\mathrm{A}+\mathrm{H} /$ hour recording did, however, correlate significantly with the polysomnography $\mathrm{A}+\mathrm{H} /$ hour asleep $(r=0.8$, $\mathrm{p}<0.001$, fig $1 \mathrm{~A})$.

When the home study scores were compared with the polysomnographic frequency of $\mathrm{A}+\mathrm{H} /$ hour in bed (median $20 \mathrm{~A}+\mathrm{H} /$ hour, range 2-50) rather than $\mathrm{A}+\mathrm{H} /$ hour asleep, the Bland-Altman plot showed no bias (fig 1C). The mean (SD) difference was - 1 (11) giving limits of agreement ${ }^{22}$ (mean difference \pm $1.96 \mathrm{SD}$ between which $95 \%$ of differences should lie) of -22 to +21 .

To obtain a criterion for diagnosis of SAHS from a home sleep study for use in the prospective trial we examined the paired results from the validation trial (fig 1A). A frequency of $>15 \mathrm{~A}+\mathrm{H} /$ hour asleep on polysomnography defines a group of patients who are known to benefit from CPAP therapy ${ }^{23}$ and is used in our laboratory as the criterion for a diagnosis of SAHS. All subjects who had a home study score of $>20 \mathrm{~A}+\mathrm{H} /$ hour recording showed $>15$ $\mathrm{A}+\mathrm{H} /$ hour asleep on polysomnography. However, the scatter of the paired results suggested that a home study diagnostic cut off of $>20$ $\mathrm{A}+\mathrm{H} /$ hour might result in false positive diagnoses when larger numbers were studied so a more conservative criterion for diagnosis of SAHS from a home study of $>30 \mathrm{~A}+\mathrm{H}$ /hour recording was adopted for use in the prospective trial.

PROSPECTIVE TRIAL

Of an initial 150 subjects (table 1) 149 completed the protocol. One subject defaulted after an unsuccessful home study. Twenty seven $(18 \%)$ home studies produced no interpretable data. In 10 cases $(7 \%)$ this was clearly due to the subject's failure to operate the equipment. Several of the other failures were due to technical problems inherent in the design of the equipment which have now been resolved. Subjects with unsuccessful studies did not differ in terms of age, BMI, or subjective daytime sleepiness (Epworth Sleepiness Scale (ESS) ${ }^{24}$ at time of initial clinic attendance) from those with successful studies, but women were overrepresented in the unsuccessful group (table 2).

One hundred and twenty three $(82 \%)$ home studies produced interpretable data (median 19 $\mathrm{A}+\mathrm{H}$ /hour recording, range 2-91); 43 (29\%) showed $>30 \mathrm{~A}+\mathrm{H} /$ hour recording confirming a diagnosis of SAHS. Of these, 40 subjects commenced CPAP therapy (characteristics in 
Table 2 Technical success or failure of home studies in the prospective trial: comparison of subject characteristics

\begin{tabular}{llll}
\hline & $\begin{array}{l}\text { Successful } \\
(n=123)\end{array}$ & $\begin{array}{l}\text { Unsuccessful } \\
(n=27)\end{array}$ & Significance \\
\hline Mean (SD) age (years) & $47(13)$ & $51(12)$ & $\mathrm{p}=0.1$ \\
Sex (\% female) & $7 \%$ & $37 \%$ & $\mathrm{p}=0.00003$ \\
Mean (SD) BMI (kg/m $\left.{ }^{2}\right)$ & $31 \quad(6)$ & $31 \quad(5)$ & $\mathrm{p}=0.7$ \\
Median (range) ESS & $10 \quad(0-23)$ & $12 \quad(0-18)$ & $\mathrm{p}=0.7$ \\
\hline
\end{tabular}

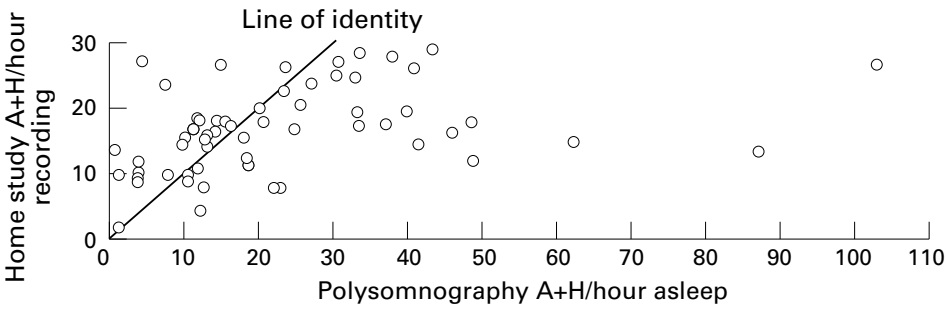

Figure 2 Prospective trial. Home sleep studies compared with polysomnographic $A+H /$ hour asleep in subjects who underwent both investigations (58 subjects with daytime sleepiness and home study score $<30 \mathrm{~A}+\mathrm{H} / \mathrm{hour})$.

Table 3 Prospective trial: comparison between home study and polysomnography results in subjects undergoing both investigations (58 subjects with daytime sleepiness and home study scores $<30 \mathrm{~A}+\mathrm{H} / \mathrm{hour}$ ). Figures are numbers of subjects

\begin{tabular}{lccccc}
\hline $\begin{array}{l}\text { Polysomnography } \\
A+\text { H/hour slept }\end{array}$ & \multicolumn{3}{l}{ Home study } & A + H/hour recording & Total \\
\cline { 2 - 5 } & $<10$ & $10-15$ & $15-20$ & $20-30$ & \\
\hline$<15$ & 9 & 6 & 8 & 3 & 26 \\
$15-30$ & 2 & 2 & 6 & 4 & 14 \\
$>30$ & 0 & 4 & 6 & 8 & 18 \\
Total & 11 & 12 & 20 & 15 & 58 \\
\hline
\end{tabular}

table 1), two were referred for tonsillectomy, and one declined treatment.

Eighty subjects had successful home studies $(54 \%)$ showing $<30 \mathrm{~A}+\mathrm{H} /$ hour recording of whom 18 (with studies showing a median of 8 $\mathrm{A}+\mathrm{H} /$ hour, range 3-19) denied troublesome daytime sleepiness (median ESS 4.5, range $0-12$ ) and four others declined further investigation. These subjects were not investigated further but were given advice appropriate to their complaints. The remaining 58 subjects (median $16 \mathrm{~A}+\mathrm{H} /$ hour, range 229) proceeded to overnight laboratory polysomnography. Including 26 whose home studies were unsuccessful, a total of 84 subjects $(56 \%)$ required polysomnography under this protocol.

Scores from polysomnography and home studies can therefore be compared in 58 subjects who, by definition, had home studies showing $<30 \mathrm{~A}+\mathrm{H} /$ hour but complained of daytime sleepiness. In this selected group the home study $\mathrm{A}+\mathrm{H} /$ hour recording and the $\mathrm{A}+\mathrm{H}$ /hour asleep from polysomnography correlated significantly $(r=0.38, \mathrm{p}<0.01$, fig 2$)$. Eighteen subjects (12\% of study population) with severe SAHS (polysomnography $>30$ $\mathrm{A}+\mathrm{H} /$ hour asleep) had home studies showing $<30 \mathrm{~A}+\mathrm{H} /$ hour recording and four $(3 \%)$ of these showed $<15 \mathrm{~A}+\mathrm{H} /$ hour recording (table 3).

COSTS AND DURATION OF INVESTIGATION

The cost of a home study was calculated as $£ 46$ and the cost of a laboratory study as $£ 210$. The mean cost and median duration of investigation in the 150 prospective trial subjects, allowing for both studies in those who required polysomnography after their home study, were lower than those of the controls (table 4). The reduction in duration of investigation was due to the avoidance of waiting times for laboratory investigation.

To see whether a lower diagnostic cut off on home study scores could have reduced the number of laboratory studies without impairing diagnostic accuracy we analysed the data from the 58 prospective trial subjects who had both home and laboratory studies. If the criterion for diagnosis of SAHS from a home study had been $>20 \mathrm{~A}+\mathrm{H} /$ hour, then 15 subjects with home study scores of 20-30 A+H/hour recording would not have undergone polysomnography and would have proceeded directly to treatment. Three of these subjects in fact had polysomnography scores of $<15$ $\mathrm{A}+\mathrm{H} /$ hour slept and have not been treated with CPAP under our standard protocols, so the reduced inconvenience to 15 patients through avoiding inpatient studies must be set against the possibly inappropriate initiation of CPAP therapy in three patients. The relative inconvenience to patients cannot be quantified, but the financial effects can be calculated. The minimum initial cost for CPAP therapy for three patients is $£ 2121$ ( $£ 707$ per patient for CPAP education, CPAP titration study, and provision of CPAP system). The financial savings from avoiding 15 laboratory studies would be $£ 3150(15 \times £ 210)$ giving a potential overall saving of $£ 1029$ or $£ 6.86$ per subject in the prospective trial.

Similarly, a diagnostic threshold of $25 \mathrm{~A}+\mathrm{H} /$ hour on home studies could have reduced the number of subjects undergoing laboratory stud-

Table 4 Final outcome of control patients, prospective trial subjects, and the subset of subjects given CPAP titration following a home study as their sole investigation

\begin{tabular}{|c|c|c|c|}
\hline & $\begin{array}{l}\text { Control patients } \\
(n=75)\end{array}$ & $\begin{array}{l}\text { All subjects } \\
(n=150)\end{array}$ & $\begin{array}{l}\text { Subjects direct to CPAP } \\
(n=40)\end{array}$ \\
\hline Median (range) days to diagnosis & $47(0-228)$ & \multirow{5}{*}{$\begin{array}{c}18(0-221) \\
(\mathrm{p}<0.001) \\
\hat{f} 164(104) \\
(\mathrm{p}<0.001) \\
92(61 \%) \\
(\mathrm{p}>0.5) \\
\quad 81(88 \%) \\
(\mathrm{p}=0.4) \\
\quad 4.7(2.4) \\
(\mathrm{p}>0.5)\end{array}$} & \multirow{5}{*}{$\begin{array}{c}\quad 7(0-98) \\
(\mathrm{p}<0.001) \\
\hat{f} 46(0) \\
(\mathrm{p}<0.001) \\
40(100 \%) \\
\text { (by definition) } \\
36(90 \%) \\
(\mathrm{p}=0.4) \\
4.9(2.3) \\
(\mathrm{p}>0.5)\end{array}$} \\
\hline Mean (SD) cost of sleep studies per patient & $£ 210(0)$ & & \\
\hline Number offered CPAP ( $\%$ of total) & $50(67 \%)$ & & \\
\hline Number continuing CPAP ( $\%$ of those offered CPAP) & $40(80 \%)$ & & \\
\hline Mean (SD) CPAP usage (hours/night) & $5.0(2.4)$ & & \\
\hline
\end{tabular}

Significance refers to difference from control group. 
ies by nine, of whom two had $<15 \mathrm{~A}+\mathrm{H} /$ hour on polysomnography and have not proceeded routinely to CPAP. The overall cost saving comes to $£ 476$ ( $£ 3.17$ per subject), again without quantifying the inconvenience to the patients.

\section{FINAL OUTCOME OF PROSPECTIVE TRIAL} SUBJECTS

When the subjects who had followed the home study protocol were compared with the control patients there was no difference in the proportion offered CPAP, the proportion continuing CPAP, or their nightly usage of CPAP. CPAP usage was also identical in the subset whose only investigation was a home study (table 4).

\section{Discussion}

This study shows that an investigative protocol based on home sleep studies can be used to diagnose SAHS with similar treatment outcome compared with a protocol using laboratory polysomnography alone and with reduced costs. There is also a benefit in reduced investigation time that is dependent on the waiting time for laboratory investigation and will vary between different centres. In our centre use of this investigative protocol reduced the demand for laboratory polysomnography by $44 \%$ and so cut the waiting time for all sleep laboratory investigations. The reduced contact with laboratory staff and loss of some opportunities for patient education did not affect the final outcome in terms of the subjects' uptake or nightly usage of CPAP. We would, however, emphasise the importance of expert technical support for explanation of the use of the portable equipment, CPAP education and mask fitting, and particularly for interpretation of the raw home study data which was all performed manually.

The validation trial shows that, in our clinic population, the Eden Trace sleep study system used at home produces a score that correlates with the polysomnographic $\mathrm{A}+\mathrm{H}$ averaged over sleep time (also known as the apnoea/ hypopnoea index, AHI) and is thus potentially usable as a diagnostic test for SAHS. However, in terms of actual agreement of recording methods rather than correlation, the variable measured during a home study $(\mathrm{A}+\mathrm{H}$ averaged over recording time) is equivalent to the polysomnographic frequency of respiratory events averaged over time spent recording, not over time asleep, as the home study gives no information about sleep time. This agreement shows wide limits, demonstrating considerable variation in both tests. The home study score is therefore not identical to the polysomnography AHI, and diagnostic criteria derived from polysomnography should be applied with caution. For the design of the prospective part of this trial the diagnostic criterion of $>30 \mathrm{~A}+\mathrm{H} /$ hour on home study which we derived from the validation trial was deliberately conservative. Any bias introduced into the prospective trial by this criterion will tend to reduce the practical benefits shown by the home studies protocol.

The prospective trial confirms the practicality of an investigative protocol based on home studies for local patients, with an acceptably low rate of technically unsuccessful studies once patients with obvious relevant disabilities are excluded. The results suggest, however, that home studies alone cannot exclude a diagnosis of SAHS in patients with daytime sleepiness. In the group most likely to present diagnostic problems - that is, those with daytime sleepiness but a low or normal home study score ( $<30 \mathrm{~A}+\mathrm{H} /$ hour recording) - the limited home studies failed to identify several subjects who had severe SAHS on polysomnography. The reason for the discrepancy is likely to be a combination of factors including the nightto-night variation in respiratory abnormality seen in any sleep study ${ }^{25}$ which is more marked in those with mild SAHS, ${ }^{26}$ the tendency of the limited home study to underestimate the frequency of sleep-related abnormalities in subjects who spend a significant part of the night awake since actual sleep time is not recorded, and the different techniques for recording respiratory variables. This illustrates the potential for diagnostic error suggested by the validation study and suggests that sleep services or research trials depending solely on home limited sleep studies may underestimate the severity of SAHS or miss the diagnosis in an important group of patients.

Debate continues as to whether CPAP treatment should be guided by the frequency of respiratory irregularity alone. Pressure for criterion based therapy from purchasers in the $\mathrm{UK}$ and evidence based approaches overseas ${ }^{27}$ are demanding strict definitions as to which SAHS patients should receive CPAP. The standard protocol in our laboratory is to offer CPAP to all those with a polysomnographic AHI of $>15 /$ hour who have troublesome sleepiness. To make a positive diagnosis of SAHS from a home study we continue to use the conservative criterion of $>30 \mathrm{~A}+\mathrm{H} /$ hour recording which was derived from the validation trial. The prospective trial indicates that a lower cut off would result in a small number of false positive diagnoses and give a small initial benefit in financial terms through reducing the number of laboratory studies. Given the inconvenience for patients of an inappropriate trial of CPAP therapy as well as the psychological and legal consequences of a false diagnosis, we have concluded that in our future practice the diagnostic criterion for home studies should not be changed.

The authors would like to thank the nursing, administrative, technical, and research staff of the Edinburgh Sleep Laboratory for assistance and support. Dr Whittle and Dr Mortimore were supported by the Wellcome Trust. We are not aware of any conflicts of interest.

\footnotetext{
1 Douglas NJ, Thomas S, Jan MA. Clinical value of polysomnography. Lancet 1992;339:347-50.

2 Phillipson EA, Remmers JE. American Thoracic Society Consensus Conference on indications and standards fo cardiopulmonary sleep studies. Am Rev Respir Dis 1989, 139:559-68.

3 McAvoy RD. Guidelines for respiratory sleep studies. Sydney:
} Thoracic Society of Australia and New Zealand, 1988. 
4 Young T, Palta M, Dempsey J, Skatrud J, Weber S, Badr $S$. The occurrence of sleep disordered breathing among middle aged adults. $N$ Engl $\mathcal{F}$ Med 1993;328:1230-5.

5 Emsellem HA, Corson WA, Rappaport BA, Hackett S, Smith LG, Hausfeld JN. Verification of sleep apnea using a portable sleep apnea screening device. South Med $\mathcal{F}$ 1990;83:748-52.

6 Redline S, Tosteson T, Boucher MA, Millman RP. Measurement of sleep-related breathing disturbances in epidemiologic studies. Chest 1991;100:1281-6.

7 Gyulay S, Gould D, Sawyer B, Pond D, Mant A, Saunders N. Evaluation of a microprocessor-based portable home monitoring system to measure breathing during sleep. Sleep 1987;10:130-42.

8 Stoohs R, Guilleminault C. Mesam 4: an ambulatory device for the detection of patients at risk for obstructive sleep apnea syndrome. Chest 1992;101:1221-7.

9 White DP, Gibb TJ, Wall JM, Westbrook PR. Assessment of accuracy and analysis time of a novel device to monitor

sleep and breathing in the home. Sleep 1995;18:115-26.
Orr WC, Eiken T, Pegram V, Jones R, Rundell OH. A laboratory validation study of a portable system for remote recording of sleep related respiratory disorders. Chest 1994; 105: $160-2$

11 Man GC, Kang BV. Validation of a portable sleep apnea monitoring device. Chest 1995;108:388-93.

12 Bearpark H, Elliott L, Grunstein R, Cullen S, Schneider $\mathrm{H}$, Althaus W, et al. Snoring and sleep apnea - a population study in Australian men. Am 7 Respir Crit Care Med 1995; 151:1459-65.

13 Ferini-Strambi L, Zucconi M, Palazzi S, Castronovo V, Oldani A, Della Marca G, et al. Snoring and nocturnal oxygen saturations in an Italian middle-aged male popuoxygen saturations in an Italian

14 Hida W, Shindoh C, Miki H, Kikuchi Y, Okabe S, Taguchi $\mathrm{O}$, et al. Prevalence of sleep apnea among Japanese industrial workers determined by a portable sleep monitoring system. Respiration 1993;60:332-7.

15 Coppola MF, Lawee M. Management of obstructive sleep apnoea syndrow in

16 de Maine J, Sandblom R, Hert R, Bloss R. Utilization and cost of evaluating obstructive sleep apnea in managed care
- effect of an integrated home and sleep lab testing clinical pathway (abstract). Am $\mathcal{F}$ Respir Crit Care Med 1996;153 A870.

17 Ninane V, Art G, Dachy B, Sergysels R. Polysomnography in the home (abstract). Am F Respir Crit Care Med 1994 149:A52.

18 Ferguson KA, Keenan SP, Love LL, Al-Majed SA, Fleetham A. Validation of home monitoring with CNS Poly G/Sleep IT in patients with obstructive sleep apnoea (abstract). $A m$ 7 Respir Crit Care Med 1994;149:A51.

19 Whyte KF, Allen MB, Jeffrey AA, Gould GA, Douglas NJ. Clinical features of the sleep apnoea/hypopnoea syndrome. $Q \mathcal{F}$ Med 1989;72:659-66.

20 Rechtshaffen A, Kales A, eds. A manual of standardised terminology, techniques and scoring systems for sleep stages of human subjects. Bethesda, MD: National Institutes of of human subjects. Bethesda, $M$

21 Gould GA, Whyte KF, Rhind GB, Airlie MA, Catterall JR, Shapiro CM, et al. The sleep hypopnoea syndrome. An Rev Respir Dis 1988;137:895-8.

22 Bland JM, Altman DG. Statistical methods for assessing agreement between two methods of clinical measurement. Lancet 1986;i:307-10.

23 Engleman HM, Martin SE, Deary IJ, Douglas NJ. The effect of continuous positive airway pressure therapy on daytime function in the sleep apnoea/hypopnoea syndrome. Lancet 1994;343:572-5.

24 Johns MW. A new method for measuring daytime sleepiness: the Epworth Sleepiness Scale. Sleep 1991;14:540-5.

25 Meyer TJ, Eveloff SE, Kline LR, Millman RP. One negative polysomnogram does not exclude obstuctive sleep apnea. 1993;103:756-60

26 Wittig RM, Romaker A, Zorick FJ, Roehrs TA, Conway WA, Roth T. Night-to-night consistency of apneas during sleep. Am Rev Respir Dis 1984;129:244-6.

27 The Australian Health Technology Advisory Committee of the National Health and Medical Research Council and the New Zealand Ministry of Health. The effectiveness and cost-effectiveness of nasal continuous positive airways pressure in the treatment of obstructive sleep apnoea in adults. Canberra, Australia: National Health and Medical Research Council, 1996. 